\title{
ON THE FORMATION OF NEGATIVELY ELECTRIFIED RAIN DROPS.
}

By Fernando SANFord.

$\mathrm{IT}^{\mathrm{T}}$ is known that in fair weather the electrical condition of the atmosphere is usually positive everywhere over the earth's surface. This is regarded as the normal condition of the atmosphere, and it is subject to periodical changes which for a given place are fairly constant, day after day and year after year. It is also well known that on account of its high specific inductive capacity water will take a positive electrical charge by contact with nearly all known substances. Lord Kelvin, Lenard and especially Coehn and Mozer ${ }^{1}$ have shown that gases bubbled through pure water carry off charges and leave the water positively electrified. This makes it practically certain that the positive electrification of the air is located upon the minute drops or the molecules of water in the air.

It has also been known for a long time that the air in the vicinity of waterfalls becomes negatively electrified, and Elster and Geitel showed that this condition may be appreciable to heights of at least 500 meters above the waterfall. Lenard ${ }^{2}$ showed that the negative electrification arises near the foot of the waterfall where the water is dashed or blown into spray, and that it seems to occur wherever in rapids or falls spray is produced, while it is imperceptible over a smoothly flowing stream.

Dr. Simpson ${ }^{3}$ showed that when drops of distilled water fall through a vertical air blast strong enough to produce spray the small drops of water formed have negative charges much more often than positive charges. Since the small drops are carried upward much faster by a rising current and since they fall more slowly through still air than the large drops, they may become separated by air currents or by gravitation from the larger electropositive drops. Since drops of water cannot fall through still air faster than about 8 meters per second without being torn to pieces by atmospheric resistance, it would seem that an electric separation must be produced whenever large raindrops fall from a considerable elevation.

${ }^{1}$ Ann. d. Phys., 43, ro48 (I9r4).

2 Ann. d. Phys., 46, 584 (1892).

${ }^{3}$ Quoted from Humphreys's article on "The Thunderstorm and Its Phenomena" in Monthly Weather Review, June, I9r4. 
Given the small negatively electrified drops that are thus blown off from the positive drops, it seems possible to account for all the phenomena of thunderstorms, and Humphreys has done this very successfully in the paper to which reference has been made. The question which seems thus far to have been impossible of explanation is how negatively electrified drops may be blown from positively electrified drops by a wind which regularly gives off positive charges to water. In order to find an answer to this question it is important to know how exretmely small drops, such as take negative charges, may be formed from larger drops.

It has frequently been observed that when a drop of liquid breaks away from a larger mass the liquid which joins the drop to the larger mass is drawn out into a narrow cylindrical neck before it is pinched off at one end by the contraction of its surface film. Plateau ${ }^{1}$ has shown that a liquid cylinder is in a condition of unstable equilibrium when its length exceeds about three $(\pi)$ times its diameter. Such a cylinder will break up into segments whose distances apart are approximately equal to the circumference of the cylinder, and these segments spontaneously take the spherical form. Accordingly, when such a liquid cylinder has been drawn out between two separating drops and is pinched off at one end by its surface film it immediately forms one or more small drops which break away from the larger drop.

The formation of these little drops between larger ones has frequently been shown photographically, but it may be observed directly without any artificial aids. Thus if ink be dropped from a pen filler which is held before an illuminated white surface, as a sheet of white paper against a windowpane, a small drop may almost invariably be seen following the larger drop. If a low power magnifying glass be placed in front of the falling drop so that it will come into the field of view just after breaking away from the dropper, the little drop may be seen to form from the liquid neck which clings to the falling drop instead of to the dropper. That is, the liquid which forms the little drop breaks away from the dropper and then later from the falling drop. The little drops may be collected and their size compared with that of the larger drop by catching them on a moving piece of paper. Since the little drop falls more slowly than the large drop it may easily be caught to one side of it. The same thing may be done by giving the dropper a horizontal motion just as the drop separates from it. When the drops are collected in this way, the large drop is seen to have several thousand times the volume of the little droplet.

If a drop of ink or of colored glycerine be dropped through a hori-

1 Statique Des Liquides, Vol. I, p. 75. 
zontal air blast, such as is used with a laboratory blow pipe, the drop will be blown to pieces if the velocity of the air is sufficiently high. By spreading sheets of paper below the falling drop the separate drops may be collected. It will then be seen that there are a few larger drops and a great many little droplets. If the blast is not too strong, the original drop still remains larger than any of the others, and the drops of intermediate size are apparently blown off from this one at a time. This conclusion may be verified by dropping a liquid from a sufficient height to cause the drops to be separated by air friction, when it will be seen that the first division is into two drops of very unequal size. Figure I shows a photograph of the splashes made on a sheet of absorbent paper by a drop of ink falling from a height of about 20

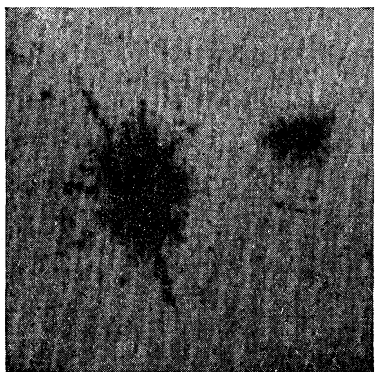

Fig. 1. feet. An observer noticed that the drop struck the paper as two separate drops, and the splashes show that one was much larger than the other.

It is plain that when the drops of intermediate size are blown off from the larger drop there are always one or more of the little droplets formed from the liquid neck which is drawn out between the two, and these tiny droplets are those which receive the negative charges. The conditions for forming the negative droplets are then pretty definitely known. First, a drop smaller than the original positively electrified drop is blown off from it. This drop is also positively electrified. Before it breaks away from the original drop, a narrow neck of water is drawn out between them. This breaks away from the original drop, then from the secondary drop, and becomes a negatively electrified droplet.

This phenomenon of charging a small body by induction between two similarly electrified bodies may easily be reproduced in the laboratory. If two insulated metal spheres or cylinders of unequal radius be placed in contact and charged positively and then be separated to a small distance while still remaining charged, a very small insulated sphere when introduced between them may take a positive charge by contact with the surface of the larger sphere or cylinder or a negative charge by contact with the smaller. Thus the inductive effect of the larger sphere upon the smaller is sufficiently great to cause it to give off a negative charge to a small conductor touched to the point nearest to the larger sphere; or to put it another way, the charge which the little conductor may take by induction between the two spheres is greater than the opposite charge which it may take from the smaller sphere while in the same position. 
The conditions under which the negatively electrified water droplets are formed seem to be exactly reproduced in this laboratory experiment. The little droplets are formed between two positively electrified drops of unequal size. They break away first from the larger drop, and then while still very close to it, from the smaller drop, taking a negative charge by induction of the larger drop.

The only reference to the above induction experiment which the present writer has been able to find in the literature of electricity is in Dr. Thomas Thomson's Heat and Electricity, which was published in r83o. Dr. Thomson attributes the discovery of the phenomenon to Coulomb. He says that by using two globes, one I I inches in diameter and the other 8 inches in diameter, which were positively charged while in contact, Coulomb was able to take a negative charge from the smaller globe when their surfaces were separated by one inch, and that when a globe 4 inches in diameter was used with the I I-inch globe, Coulomb was able to take a negative charge from the smaller globe when they were two inches apart.

STANFORD UNIVERSITY, January 22, I9I8. 


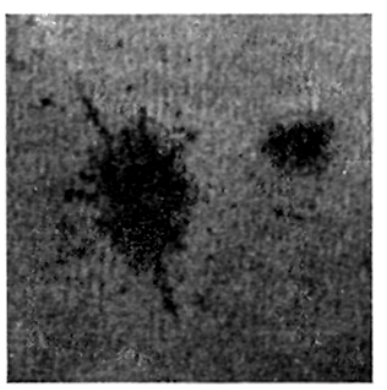

Fig. 1. 